\title{
A Deep Convolutional Neural Network Architecture for Cancer Diagnosis using Histopathological Images
}

\author{
Karthika Gidijala, Mansa Devi Pappu, Manasa Vavilapalli, Mahesh Kothuru
}

\begin{abstract}
Many different models of Convolution Neural Networks exist in the Deep Learning studies. The application and prudence of the algorithms is known only when they are implemented with strong datasets. The histopathological images of breast cancer are considered as to have much number of haphazard structures and textures. Dealing with such images is a challenging issue in deep learning. Working on wet labs and in coherence to the results many research have blogged with novel annotations in the research. In this paper, we are presenting a model that can work efficiently on the raw images with different resolutions and alleviating with the problems of the presence of the structures and textures. The proposed model achieves considerably good results useful for decision making in cancer diagnosis.
\end{abstract}

Keywords: Histopathological images of breast cancer, Deep Learning, CNN.

\section{INTRODUCTION}

The massive growth of breast cancer and its incidence in the recent years, death rate has been considerably reduced in most developed countries. Many breakthroughs are notified in early detection and screening methods through medical imaging and analysis. Classification of patients with cancer or no-cancer using clinical records requires careful scrutiny with high sensitivity and specificity in a diagnostic test. The core of the consensus in breast cancer research deals with study of multiple magnification levels of histopathological images, which is time-hard and paves into multiple theses of analyses. Though objectively, the assuages on saving patients life, the research experts rely on sophisticated Computer Aided Diagnosis (CAD) systems. The in vitro golden source of datasets are collected in the process for decision making with factorized right time, right diagnosis.

Manuscript received on September 29, 2021.

Revised Manuscript received on October 05, 2021.

Manuscript published on October 30, 2021.

* Correspondence Author

Karthika Gidijala*, Department of Computer Science and Engineering, GITAM Institute of Technology, GITAM Deemed to be University, Visakhapatnam, India. Email: kgidijal@gitam.edu

Mansa Devi Pappu, Department of Computer Science and Engineering, GITAM Institute of Technology, GITAM Deemed to be University, Visakhapatnam, India. Email: mpappu@gitam.edu

Manasa Vavilapalli, Department of Computer Science and Engineering, Dadi Institute of Engineering and Technology, Visakhapatnam, India. Email: manasa@diet.edu.in

Mahesh Kothuru, Department of Computer Science and Engineering, GITAM Institute of Technology, GITAM Deemed University, Visakhapatnam, India. Email: mkothuru@gitam.edu

(C) The Authors. Published by Blue Eyes Intelligence Engineering and Sciences Publication (BEIESP). This is an open access article under the CC BY-NC-ND license (http://creativecommons.org/licenses/by-nc-nd/4.0/)
The theoretical annotations built helps experts in the studies and directing situations into prospective decision making. Automated systems promise to be more accurate and efficient that can reduce variability in diagnosticapplications and increase prospects of histopathological diagnoses.

\section{LITERATURE REVIEW}

The core processing of analysis and classification of breast cancer images is vested into two stages: Pre-processing and preparation using Image Processing, Analysis and Classification Deep Learning (Convolution Neural Networks). The objective of histopathology is to distinguish normal tissue with a malign tissue to enable prognostic evaluation [1]. The maligning of breast tissue is carcinoma, where the morphology of the tissue cells determines the seriousness of malignity [2]. A combination of haematoxylin and eosin ( $\mathrm{H} \& \mathrm{E})$ is noted to be as the stain of tissue specimen for routine histopathological diagnoses. Digital pathology image analysis works on rule-based methods to machine learning and deep learning methods. The automated end-to-end processing is absolutely achieved with the implementations of convolution neural networks, particularly in the domain medical image analysis A pretrained CNN model available on ImageNet with gradient boosting algorithm is employed on $\mathrm{H} \& \mathrm{E}$ stained histopathological breast cancer images [3]. Even fully operated Computer Aided Diagnostic systems use deep learning and machine learning algorithms, a CAD system for breast cancer diagnosis is described in [5], detects and extracts patches containing masses and classifies them without any external user intervention and without preprocessing of pectoral muscles removal or masses segmentation. Inherent Features and Relationships in data can be learnt with machine learning, for an image data convolution neural networks have been proved as efficient hallmark that support intelligent decision making. Convolution neural networks can solve critical problems of decision making on spatial and temporal aspects of image data [6]. A considerate inter-pathologist-variability exists in traditional histopathological systems, which can be mitigated quantitative analysis, where the processes are too time consuming in clinical setups [7]. Variability in appearance due to hematoxylin and eosin stained areas is one of the major research interest to prove accurate image analysis.

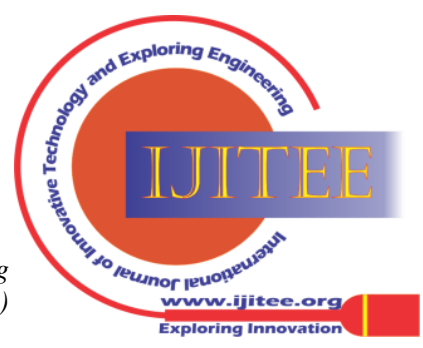


The sophisticated imaging devices produce enormous sizes of images with raw representation of image components, with large dimensions occupying large storage space, viz whole-slide digital pathology images (WSIs). In the field of digital pathology, the assessment from whole diagnostic images (WSIs) presents several particular difficulties. The pictures are very big and must be subdivided into 1000s of tiny tiles before they're even handled in any way at all. When performing a job, it is possible that both the backdrop at different magnifications and the detail at various magnifications are relevant; thus, information from different scales must be combined. The challenges in working with such images is to break the barrier of handling large images and the errors cue to H\&E. Convolutional Neural networks can face this challenge of uncertainty of accuracy, size and formats of images. In the early days of artificial intelligence Robertson et. al [8] has proved that AI can mitigate with the barriers of the image qualities and problem of identification of tumors can recede drastically with the implementation of machine learning. Noteworthy annotations have been made in the consensus to the development of methods in classification and recognition of histological images of breast cancer.

\section{RELATED WORK}

For developing Computer Aided Diagnosis systems (CADs), many breast cancer datasets exist. These datasets use both deep learning and conventional models. Though most of these resources have different trade-offs for practitioners, it's important to understand the relative availability or internal clinical worth of the data before proceeding. Leveraging state-of-the-art computer vision and machine learning techniques, automated examination of pathology specimens may be expedited, which, in turn, cuts down the analysis time. We can report that the most up-to-date and clinically relevant public breast cancer histopathology dataset available to date is Break His.

Histopathological Categorization of Breast Cancer

\begin{tabular}{|l|}
\hline Benign \\
\hline
\end{tabular}

$\begin{aligned} \text { Adenosis (A) } & \text { (DC) Ductal Carcinoma } \\ \text { Fibroadenoma (F) } & \text { (LC) Lobular Carcinoma } \\ \text { Phyllodes Tumor (PT) } & \text { (MC) Mucinous Carcinoma } \\ \text { Tubular Adenoma (TA) } & \text { (PC) Papillary Carcinoma }\end{aligned}$

Fig. 1 Classification of Breast Cancer

Images of various tumors and benign/malignant conditions are grouped by degree of magnification into four separate arrays, which include labels for major categories (benign/malignant) and subcategories (A/F/PT/TA/PC/DC/LC/MC).

Pictures are labelled as benign or malignant to assist the user in distinguishing between the two types of images. Images are further subdivided into the following eight categories to further assist the user: In the case of benign pictures, Adenosis (A), Fibroadenoma (F), Phyllodes Tumor (PT), and Tubular Adenoma (TA) are used, whereas Ductal
Carcinoma (DC), Lobular Carcinoma (LC), Mucinous Carcinoma (MC), and Papillary Carcinoma (PC) are used in the case of malignant images. The different features that this statement possesses allow problem solvers to re-work this situation in the following way: binary classifiers can correctly identify whether an input layer is safe or dangerous, while multi-category classifiers can determine the specific class belonging to (A/F/TA/PT/DC/LC/MC/PC) classes. The structure makes it possible for practitioners to handle this issue, whether as a binary classification job with a magnification dependent training method or as a multicategory classification task with an independent training strategy. On each supervised learning, the adopted model employs one of two approaches: one that makes use of a magnification-specific training scenario, or one that does not. The magnification-specific method includes training four different models on different magnification subsets, resulting in eight models total. When training a unique model with all magnification levels included, in the magnification-independent method, the process is known as a data abstraction since all magnification levels are simply lumped together.

\section{FEATURE EXTRACTION}

Many methods exist in the research to extract robust features of images. In general features of images are constituent building blocks of image, viz.:edges, corners, blobs, clouds, ridges, etc. A feature is an intended property of the process that is undertaken for analyses in a given domain of study. A feature syntactically comprises of attributes. Attributes are properties of pixels at the physical view of an image. Scientific methods are applied to build a deep learning model, which could be used to extract features from the images, generally include histogram based methods, matrix based methods, binary pattern methods. Pathological properties required may also be extracted from the computations and algorithms of image processing and deep learning, which may become input to the future experimentations in wet lab. Recent research literature exposes experiments on breast cancer images. Most of the experiments take a reference image that contains a healthy image of breast and the floating image containing tumor is mapped with the symptoms of the healthy. Some of the areas of the images are tested with statistically known similarity functions such as difference of squares, imageratio uniformity, cross correlations, phase correlations and mutual information about the selected areas. A set of feature points from each reference and floating images are considered extracted and similarity is tested[1]. Transformation parameters are analyzed and the structure of the cytoplasm (tumor), points of bifurcation, cross-over points and branching are studied by extracting algorithmically and as well in wet labs.

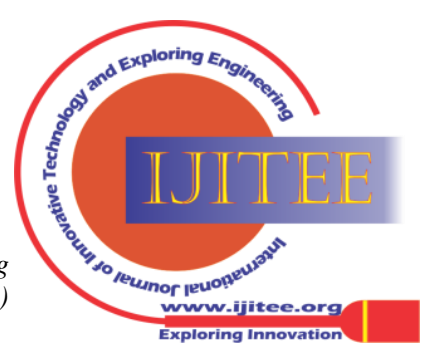




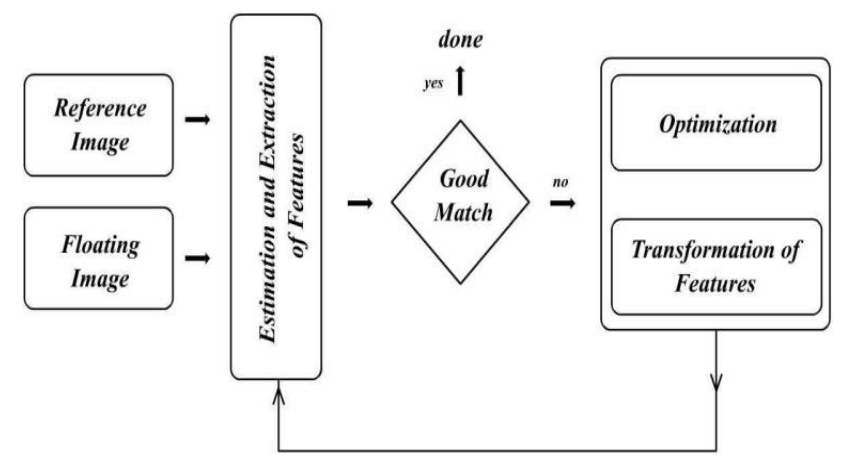

Fig. 2 Basic Image Analysis Framework

In general components of interest in breast cancer such as cytoplasm or nuclei are not clearly visible, due to grayscale aberrations. Therefore, a crucial procedure like tissue staining is implemented just before visualization. Thus, staining helps to highlight each component for better morphological analyses under the sophisticated microscope.

\section{A. DEEP LEARNING METHODOLOGY}

The images of the breast cancer are collected and scaled into different sizes. This process of phasing of the breast images reduces the learning time; therefore the insignificant portions are eliminated from learning. The collected images are in the gray-scale, conflicting brightness intrudes the process of detecting the presence of tumour. The shape of the tumour shall be the source of identification of benignity or malignity in the images. To implement, the phased images are trained and tested into benign and malign classes.
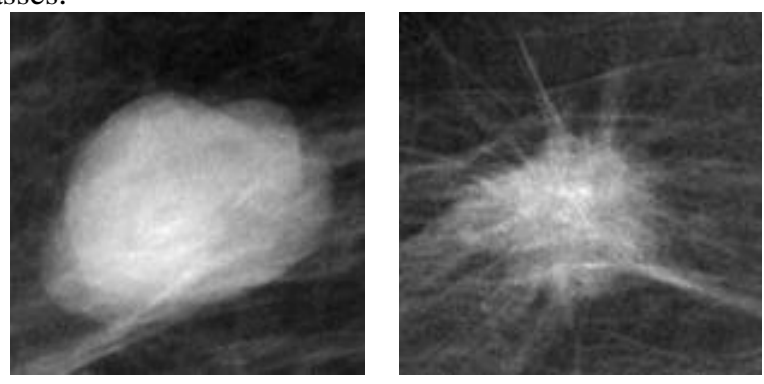

Fig. 3: Samples of mammography mass lesions; (a) benign; (b) malignant

\section{EXPERIMENTAL RESULTS}

A Deep CNN architecture is implemented to unveil the important aspects of the malignity related pathological symptoms. Into a typical DCNN architecture, an input of breast image of size $220 \times 220$ is supplied as tensor of size $192 \times$ 192. The first convolution of the specific configuration might take as $3 \times 3 \times 2$ kernel filters with stride $1 \times 1$ and a total of 24 filters are applied.

Convolution 1 produces max-pooled using a pooling layer with a stride of $2 \times 2$, resulting in half the size $96 \times 96$ of the input. After ReLU, the resulting output of the process is sent through a convolutional layer with nonlinearity. The output is then given as an input to the next convolutional layer.

In next layer 3 x 3 x 24 kernel filters with duration 1 x 1 and a total of 48 filtration are applied in the subsequent convolution layer. The second convolution operation reduced the input size to half of its size $48 \times 48$, after which the output was then scaled using a max-pooling layer with stride $2 \times 2$. Nonlinearity is added to the output of the previous convolution layer, after which it is sent into the next convolution layer, resulting in an imbibed nonlinearity. Third Layer 3x3x96 kernel filters with stride 1x1 are used in the third convolution layer. When the third convolution operation is applied, the input is "pooled" (reduced in size) using a pooling layer that has a stride of $2 \times 2$, resulting in a lower-resolution input of half the original size of $24 \times 24$. Nonlinearity is imbibed in the ReLU activation function, which is carried through to the fourth convolution layer with 192 filters and a $3 \times 3 \times 192$ kernel size.

To fill the space left behind by the reduction in the output, the image is max-pooled to a size of $12 \times 12$. In order to process the results of all of the pre-configured layers, including the activation results from ReLU, with 240 filters, a fifth convolution layer with 240 filters is fed the activation from ReLU, and a kernel size of $12 \times 12 \times 240$ is used in order to accept all of the results from previously configured layers and maximise the pooling from that layer with stride of $2 \times 2$. The tensor's resultant form now is $6 \times 6 \times 240$. So, to re-express this in a different way, the tensor is flattened and reshaped into a linear unit where the values within the neurons reflect the symptoms of malignant tissues.

In order to ensure that the network is not an overfit in learning, drop out values are used in the dropout layer. The count of neurons is reduced to the sets of classes defined to substantiate the fully connected layers of the network.

The final result of all convolutions with fully connected layers is a tensor with 48 neurons converted to the number of classes such as healthy or defective cancer image.

During the study of the analyses the outcome of the learning using the CNN with varied sized sets of filters and pooling mechanisms and subsequently filters used in the ReLU derives the observations and advantages of learning based on the inputs and the number of iterations. The question of accuracy levels obtained by number of inputs and the number of iterations is plotted graphically using epoch graph. Epoch graphs measures the adolescence of the network in learning. Epoch graph demonstrates the loss of learning in training during the process and advantage of obtaining the accuracy during the process.

Accuracy and Epoch graphs
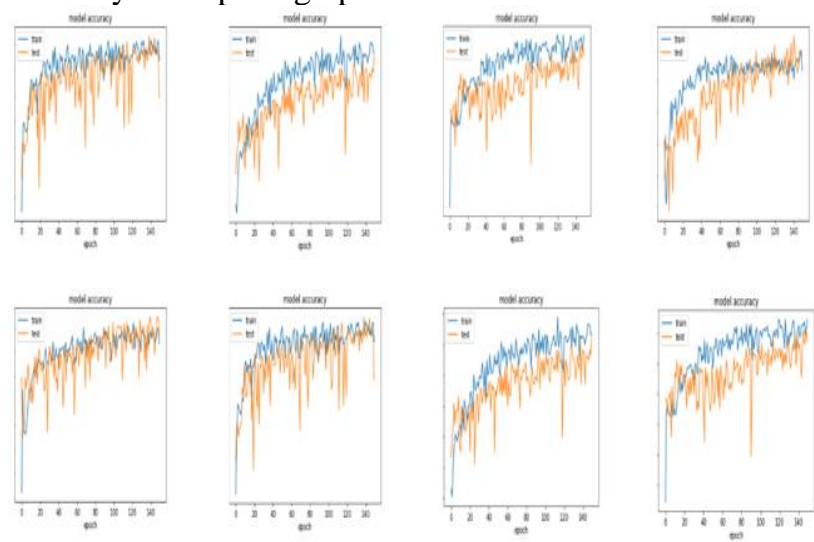

Loss and Epoch graphs

Published By:

Blue Eyes Intelligence Engineering and Sciences Publication (BEIESP)

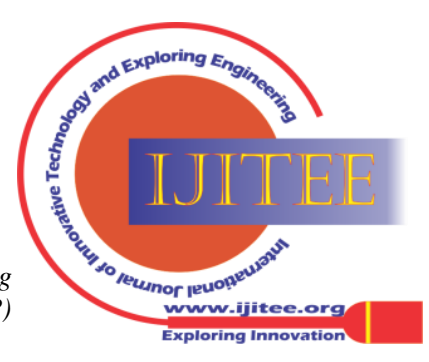



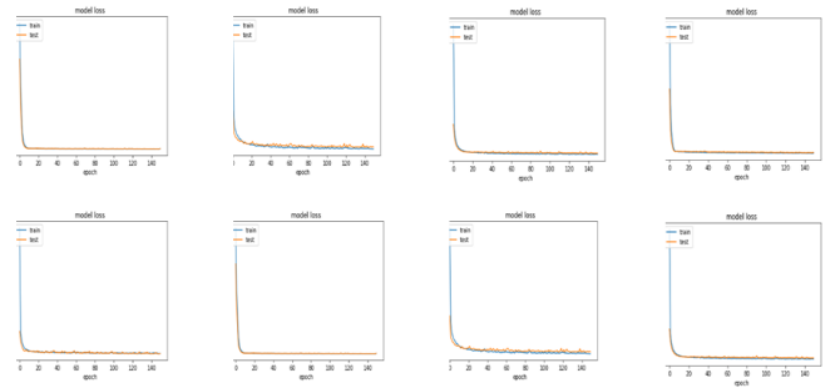

Fig. 4: The Loss and Accuracy Epoch graphs generated in Keras using a DCNN detecting the symptoms of breast cancer.

In studies using synthetic images, the amount of information gained is defined by the mean squared error, and as the risk of recurrence rises, the failure value for learning is reduced as much as feasible until it is near to zero. A graphical depiction of the CNN's academic achievement is given below the text. Each of the 100 synthetic pictures utilised in this study has a flaw, with 70 of them being used for training while about 40 being healthy and the other 30 being defective. For testing, the first 30 pictures are utilised, with 20 of them being healthy and 10 of them being faulty. As tensors are input into the network, image training is done repeatedly until the network is trained. Every image is subjected to a predetermined number of cycles: 250, with a learning rate of 0.0003 defined for each iteration.

The following experiment is carried out on the Linked list of DCNN with the help of Keras. The observations of the observatory are shown in the following diagram. Throughout each epoch, the test is carried with 250 samples, and the network is tested using 150 epochs during the whole experiment. The training and evaluation of pictures sent to the proposed network are shown in the accompanying graphs. The proposed network was found to have substantial loss and accuracy in both the training and testing phases.

The following experiment is carried out on the Flow graph of DCNN with the help of Keras. The observations of the observatory are shown in the following diagram. Throughout each epoch, the experiment is conducted with 250 samples, and the network is trained and tested using 150 epochs during the whole experiment.

The following graphs show the training and validation of images given to the proposed network, which has been arrived with significant loss and accuracy in training and validation.
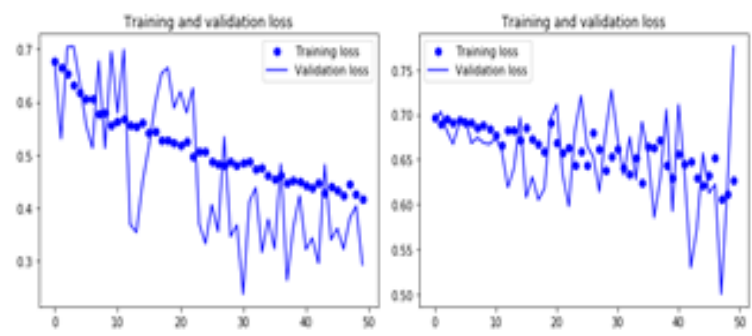

Fig. 5: Graphs obtained showing the training and validation loss due to misclassified histopathological breast cancer images, where images do not contain symptoms of cancer/tumor or contain the symptoms very feebly.
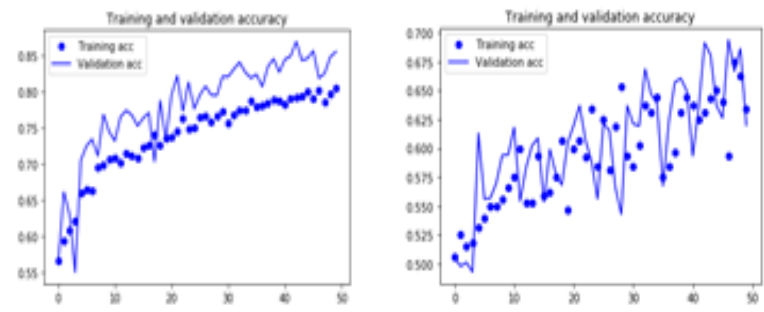

Fig. 6: Graphs obtained showing the training and validation efficiency in histopathological breast cancer images, where images contain strong symptoms of cancer/tumor.

Finally, we outline the following drawbacks of the present deep learning artificial intelligence system. Because of a possible conflict of interest, grading errors in the pictures may occur, which may have a negative impact on the model's generalisation capacity. Experimentations have been conducted on the classification of the malign categories of breast cancer DC, LC, MC, PC. In detecting the malignities the proposed CNN has succeed in achieving the accuracy. The following ROC curves have been generated on the samples of normal distribution of histopathological images for ductal, lobular, mucinous and papillary carcinoma.

Table 2: Performance of CNN for histopathological images with benign characteristics

\begin{tabular}{|r|r|r|r|r|c|c|c|}
\cline { 2 - 6 } \multicolumn{1}{c|}{} & \multicolumn{2}{c|}{ Observed } & \multicolumn{2}{c|}{ Cumulative } & \multicolumn{1}{c|}{} \\
\hline Benigntiy & TRUE & FALSE & TRUE & FALSE & FPR & TPR & AUC \\
\hline & & & 0 & 0 & 1.000000 & 1.000000 & 0.064516 \\
\hline 1 & 34 & 3 & 34 & 3 & 0.935484 & 0.989247 & 0.118259 \\
\hline 2 & 63 & 7 & 97 & 10 & 0.815939 & 0.964158 & 0.160998 \\
\hline 3 & 88 & 11 & 185 & 21 & 0.648956 & 0.924731 & 0.184244 \\
\hline 4 & 105 & 14 & 290 & 35 & 0.449715 & 0.874552 & 0.204117 \\
\hline 5 & 123 & 23 & 413 & 58 & 0.216319 & 0.792115 & 0.142791 \\
\hline 6 & 95 & 60 & 508 & 118 & 0.036053 & 0.577061 & 0.009855 \\
\hline 7 & 9 & 75 & 517 & 193 & 0.018975 & 0.308244 & 0.003509 \\
\hline 8 & 6 & 41 & 523 & 234 & 0.007590 & 0.161290 & 0.001224 \\
\hline 9 & 4 & 30 & 527 & 264 & 0.000000 & 0.053763 & 0.000000 \\
\hline 10 & 0 & 15 & 527 & 279 & 0.000000 & 0.000000 & 0.000000 \\
\hline & 527 & 279 & & & & & 0.889515 \\
\hline
\end{tabular}

The above table shows the observed and cumulative collections of histopathological images tested for benignity. The ROC curve is plotted for the above data and the AUC achieved 0.889515 , that is almost 88 percent of the images lease the features of benignity.

$\begin{array}{rll}\text { Accuracy } & (\text { ACC })= & 0.7866 \\ \text { Sensitivity } & (\text { TPR })= & 0.7921 \\ \text { Specificity } & (\text { TNR })= & 0.7837 \\ \text { False Positive Ratio } & (\text { FPR })= & 0.2163 \\ \text { Positive Predictive value } & (\text { PPV })= & 0.6597 \\ \text { Negative Predictive Value } & (\text { NPV })= & 0.8769\end{array}$

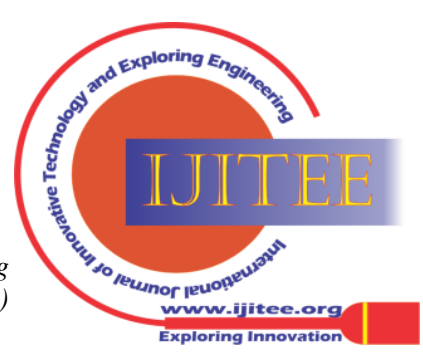




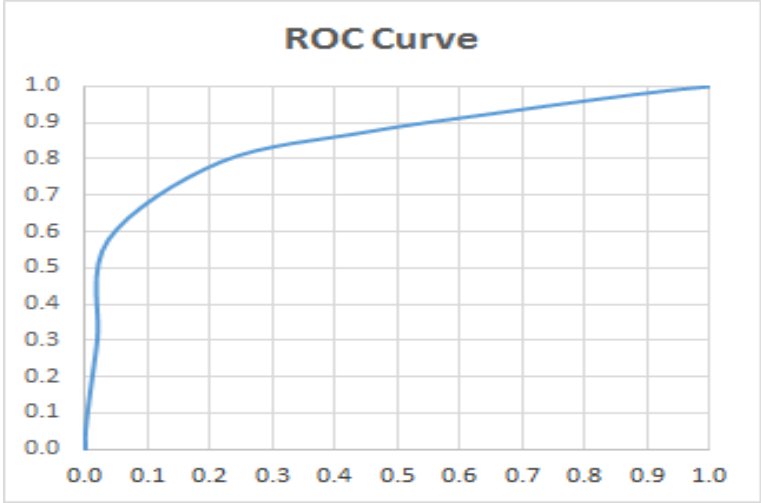

Fig. 5: ROC curve demonstrating the AUC for benign breast cancer images.

Table 3: Performance of CNN for histopathological images with malign characteristics

\begin{tabular}{|c|c|c|c|c|c|c|c|}
\hline \multirow{3}{*}{ Malignity } & \multirow{2}{*}{\multicolumn{2}{|c|}{ Observed }} & \multirow{2}{*}{\multicolumn{2}{|c|}{ Cumulative }} & \multirow[b]{3}{*}{$F P R$} & \multirow[b]{3}{*}{$T P R$} & \multirow[b]{3}{*}{$A \cup C$} \\
\hline & & & & & & & \\
\hline & TRUE & FALSE & TRUE & FALSE & & & \\
\hline & & & 0 & 0 & 1.000000 & 1.000000 & 0.082437 \\
\hline 1 & 46 & 6 & 46 & 6 & 0.917563 & 0.981013 & 0.126582 \\
\hline 2 & 72 & 8 & 118 & 14 & 0.788530 & 0.955696 & 0.157570 \\
\hline 3 & 92 & 12 & 210 & 26 & 0.623656 & 0.917722 & 0.177624 \\
\hline 4 & 108 & 15 & 318 & 41 & 0.430108 & 0.870253 & 0.185592 \\
\hline 5 & 119 & 26 & 437 & 67 & 0.216846 & 0.787975 & 0.132741 \\
\hline 6 & 94 & 68 & 531 & 135 & 0.048387 & 0.572785 & 0.013344 \\
\hline 7 & 13 & 78 & 544 & 213 & 0.025090 & 0.325949 & 0.004673 \\
\hline 8 & 8 & 52 & 552 & 265 & 0.010753 & 0.161392 & 0.001735 \\
\hline 9 & 6 & 32 & 558 & 297 & 0.000000 & 0.060127 & 0.000000 \\
\hline \multirow[t]{2}{*}{10} & 0 & 19 & 558 & 316 & 0.000000 & 0.000000 & 0.000000 \\
\hline & 558 & 316 & & & & & 0.882299 \\
\hline
\end{tabular}

The above table shows the observed and cumulative collections of histopathological images tested for malignity. The ROC curve is plotted for the above data and the AUC achieved 0.882299 , that is almost 88 percent of the images lease the features of malignity.

$\begin{array}{rll}\text { Accuracy } & (\text { ACC })= & 0.7849 \\ \text { Sensitivity } & (\text { TPR })= & 0.7880 \\ \text { Specificity } & (\text { TNR })= & 0.7832 \\ \text { False Positive Ratio } & (\mathrm{FPR})= & 0.2168 \\ \text { Positive Predictive value } & (\mathrm{PPV})= & 0.6730 \\ \text { Negative Predictive Value } & (\mathrm{NPV})= & 0.8671\end{array}$

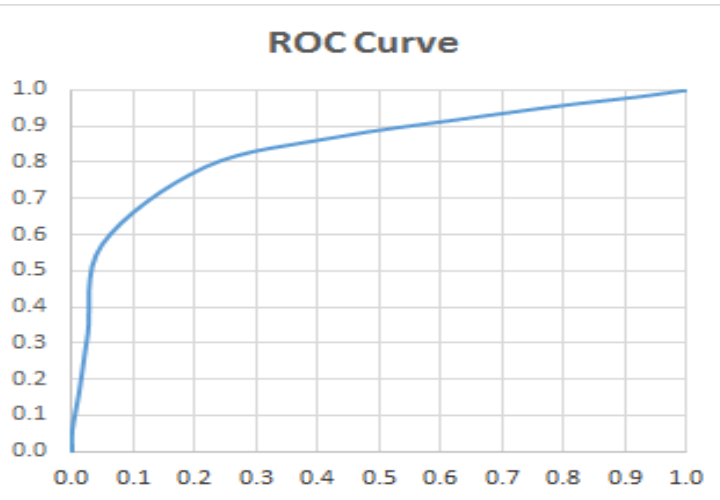

Fig. 6: ROC curve demonstrating the AUC for malign breast cancer images.
It has been found that the model achieves elegant metric performance when the histological pictures are scaled with various dimensions in order to identify malignancy in the case of Toto (Toto). Despite this, the main goal of the study is to identify malignant pictures among all of the histopathological images that have been gathered and analysed at different resolutions. The described CNN model is built in such a way that malignant pictures with a resolution of $240 \times 240$ pixels may be fed into the network, as well as convolution and pooling layers with activations in the ReLU layers. The model is built in Python using Tensor Flow, Keras in Anaconda Navigator 3, and Jupyter, and it is very adaptable. Further, in order to demonstrate the efficacy of the suggested approach, the experiment is carried out in a simple GPU setup, taking into consideration the Google Colab with a single 12GB NVIDIA Tesla K80 GPU as an example.

\section{CONCLUSION}

Through the use of various sized pictures and phasing out many irrelevant regions of the images, we presented a conventional deep CNN model with four layers that can readily identify the histological images of breast cancer in this article. In order to understand the general structures and textural characteristics of breast cancer pictures with various resolutions at different layers, the aforementioned network has been trained. The specificity and sensitivity of the model created for various instances of histological breast cancer pictures were much higher than expected, which was a significant challenge. Consequently, we conclude that this model may be readily adopted with little economic intervention by pathologists who operate in the area of cancer diagnosis and treatment planning. We recommend more qualitative feature maps may be used to test this model to find the accurate results, which would fetch real time advantages for the pathological wet labs even.

\section{REFERENCES}

1. Elston, Christopher W., and Ian O. Ellis. "Pathological prognostic factors in breast cancer. I. The value of histological grade in breast cancer: experience from a large study with long-term follow-up." Histopathology 19.5, Wiley Online Library (1991): 403-410.

2. Robertson, Stephanie, et al. "Digital image analysis in breast pathology-from image processing techniques to artificial intelligence." Translational Research 194, Elsevier (2018): 19-35.

3. Rakhlin, et al. "Deep convolutional neural networks for breast cancer histology image analysis", International conference image analysis and recognition, pp. 737- 744. Springer, Cham, 2018.

4. Spanhol, et al. "A dataset for breast cancer histopathological image classification." IEEE Transactions on Biomedical Engineering 63 , No. 7 (2015): 1455-1462.

5. Shayma'a, et. al. "Breast cancer masses classification using deep convolutional neural networks and transfer learning." Multimedia Tools and Applications 79, no. 41 (2020): 30735-30768.

6. Khan et. al. "A survey of the recent architectures of deep convolutional neural networks." Artificial Intelligence Review 53, no. 8 (2020): 5455-5516.

7. Srinidhi et. al. "Deep neural network models for computational histopathology: A survey." Medical Image Analysis (2020): 101813.

8. Robertson, S et. al. "Digital image analysis in breast pathologyFrom image processing techniques to artificial intelligence". Transl. Res. 2018, 194, 19-35.

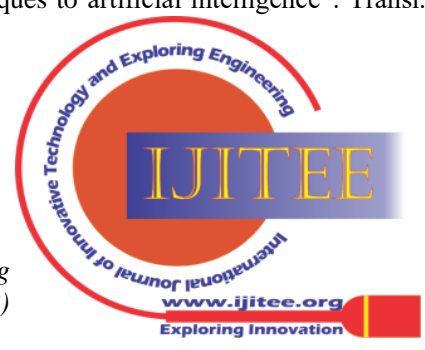




\section{AUTHORS PROFILE}

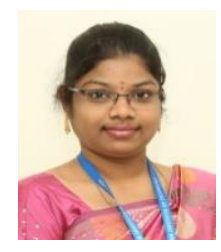

Karthika Gidijala, she received her Engineering from Kaushik college of Engg, Department of Information Technology in 2012, M.Tech from Vignan Institute of Technology, Department of Computer Science and Engineering in 2015, Started her research work in GITAM since 2018, where she now works as Assistant Professor. Her research area is on Data Mining, Machine Learning, Deep Learning.

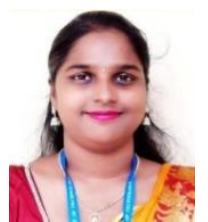

Mansa Devi Pappu, She received her Engineering from Lendi institute of Technology, Department of Computer science and Engineering in 2014, M.Tech from the GITAM, Department of Computer Science and Technology in 2016, started his research work in GITAM, since 2019, Present working as Assistant Professor in GITAM UNIVERSITY. Her research area is on Deep learning, Computer Networks.

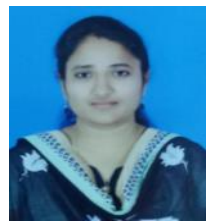

Manasa Vavilapalli, she received her Engineering from Vignan institute Of Engineering For Women, Department of Information Technology in 2012, M.Tech from GITAM (Deemed to be University), Department of Computer Science and Technology in 2014, started her research work in GITAM since 2019, she works as an Assistant Professor in Dadi Institute Of Engineering And Technology. Her research area is Computer Networks, Adhoc and sensor networks.

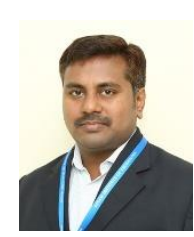

Mahesh Kothuru, He received his Engineering from MVGR College of Engg., Department of Computer Science and Engineering in 2009, M.Tech. from the Andhra University, Department of Computer Science and Systems Engineering in 2014, Started his research work in GITAM since 2018, where he now works as Assistant Professor. His research area is on Deep Learning, Computer Networks, Cloud Computing and Internet of Things.

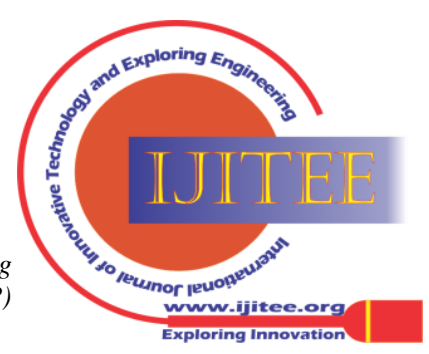

